\title{
Self-Consistent Sources and Conservation Laws for Super Tu Equation Hierarchy
}

\author{
Sixing Tao \\ School of Mathematics and Information Science, Shangqiu Normal University, Shangqiu, China \\ Email: taosixing@163.com
}

Received December 2014

\begin{abstract}
Based upon the basis of Lie super algebra $B(0,1)$, the super Tu equation hierarchy with self-consistent sources was presented. Furthermore, the infinite conservation laws of above hierarchy were given.
\end{abstract}

\section{Keywords}

\section{Super Tu Hierarchy, Self-Consistent Sources, Conservation Laws, Lie Super Algebra}

\section{Introduction}

Soliton equations with self-consistent sources have been receiving growing attention in recent years. Physically, the sources may result in solitary waves with a non-constant velocity and therefore lead to a variety of dynamics of physical models. For applications, these kinds of systems can be used to describe interactions between different solitary waves. Ma and Strampp systematically applied explicit symmetry constraint and binary nonlinearization of Lax pairs for generating soliton equation with sources [1]. Then, Ma presented the soliton solutions of the Schrödinger equation with self-consistent sources [2]. The discrete case of using variational derivatives in generating sources was discussed in [3].

With the development of soliton theory, super integrable systems associated with fermi variables have been receiving growing attention. Various methods have been developed to search for new super integrable systems, Lax pairs, soliton solutions, symmetries and conservation laws, etc. [4]-[11]. In 1997, Hu proposed the supertrace identity and applied it to establish the super Hamiltonian structures of super-integrable systems [4]. Then Professor Ma gave a systematic proof of super trace identity and presented the super Hamiltonian structures of super AKNS hierarchy and super Dirac hierarchy for application [5]. The super Tu hierarchy and its super-Hamiltonian structure was considered [6]. Recently, Yu et al. considered the binary nonlinearization of the super AKNS hierarchy under an implicit symmetry constraint [7] and the Bargmann symmetry constraint and binary nonlinearization of the super Dirac systems [8]. Meanwhile, various systematic methods have been developed to obtain exact solutions of the super integrable such as the inverse transformations, the Bäcklund and Darboux transformations, the bilinear transformation of Hirota and others [9]-[11].

This paper is organized as follows. In Section 2, the method for establishing super integrable soliton hierarchy with self-consistent sources by using Lie super algebra $B(0,1)$ was presented. For application, the super Tu hierarchy with self-consistent sources was obtained in Section 3. In Section 4, conservation laws of super Tu 
hierarchy were given.

\section{A Kind of Super Integrable Soliton with Hierarchy Self-Consistent Sources}

In the following. Consider a basis of Lie super algebra $B(0,1)$ [5]

$$
e_{1}=\left(\begin{array}{ccc}
1 & 0 & 0 \\
0 & -1 & 0 \\
0 & 0 & 0
\end{array}\right), \quad e_{2}=\left(\begin{array}{lll}
0 & 1 & 0 \\
1 & 0 & 0 \\
0 & 0 & 0
\end{array}\right), \quad e_{3}=\left(\begin{array}{ccc}
0 & 1 & 0 \\
-1 & 0 & 0 \\
0 & 0 & 0
\end{array}\right), \quad e_{4}=\left(\begin{array}{ccc}
0 & 0 & 1 \\
0 & 0 & 0 \\
0 & -1 & 0
\end{array}\right), \quad e_{5}=\left(\begin{array}{lll}
0 & 0 & 0 \\
0 & 0 & 1 \\
1 & 0 & 0
\end{array}\right) .
$$

We introduce the loop algebra $\tilde{B}(0,1)$ as follows

$$
\tilde{B}(0,1)=\{A \mid A \in R(\lambda) \otimes B(0,1)\}
$$

where the loop algebra $\tilde{B}(0,1)$ is defined by $\left\{\lambda^{n} A \mid n \geq 0, A \in B(0,1)\right\}$.

Consider the auxiliary linear problem

$$
\left(\begin{array}{l}
\phi_{1} \\
\phi_{2} \\
\phi_{3}
\end{array}\right)_{x}=U(u, \lambda)\left(\begin{array}{l}
\phi_{1} \\
\phi_{2} \\
\phi_{3}
\end{array}\right), \quad U(u, \lambda)=e_{0}(\lambda)+\sum_{i=1}^{5} u_{i} e_{i}(\lambda), \quad\left(\begin{array}{l}
\phi_{1} \\
\phi_{2} \\
\phi_{3}
\end{array}\right)_{t_{n}}=V(u, \lambda)\left(\begin{array}{l}
\phi_{1} \\
\phi_{2} \\
\phi_{3}
\end{array}\right),
$$

where $u=\left(u_{1}, \cdots, u_{s}\right)^{\mathrm{T}}, U(u, \lambda)=u_{1} e_{1}+\cdots+u_{p} e_{p}, u_{i}=u_{i}(x, t) \quad(i=1,2, \cdots, p), \quad \phi_{i}=\phi_{i}(x, t)$ are field variables defining on $x \in R, t \in R, e_{i}(\lambda)=e_{i}(x, t, \lambda) \in \tilde{B}(0,1)$.

From the spectral problem (3), the compatibility condition gives rise to the well-known zero curvature equation

$$
U_{t_{n}}-V_{x}+[U, V]=0, \quad n=1,2, \cdots
$$

The general scheme of searching for the consistent $V^{(n)}$ and generating a hierarchy of nonlinear equations was proposed as follows [5]. We solve the equation

$$
V_{x}=[U, V], \quad V=\sum_{m=0}^{\infty} V_{m} \lambda^{-m}=\sum_{m=0}^{\infty} \lambda^{-m}\left(\begin{array}{ccc}
A_{m} & B_{m}+C_{m} & \rho_{m} \\
B_{m}-C_{m} & -A_{m} & \delta_{m} \\
\delta_{m} & -\rho_{m} & 0
\end{array}\right),
$$

And search for $\Delta_{n}(u, \lambda) \in \tilde{B}(0,1)$, such that $V^{(n)}$ can be constructed by

$$
V^{(n)}=\sum_{m=0}^{n} V_{m} \lambda^{n-m}+\Delta_{n}(u, \lambda), \quad \Delta_{n}(u, \lambda)=\left(\begin{array}{ccc}
\Delta_{n 1} & \Delta_{n 2}+\Delta_{n 3} & \Delta_{n 4} \\
\Delta_{n 2}-\Delta_{n 3} & -\Delta_{n 1} & \Delta_{n 5} \\
\Delta_{n 5} & -\Delta_{n 4} & 0
\end{array}\right)
$$

where $\Delta_{n i}(1 \leq i \leq 5)$ are linear functions of $A_{m}, B_{m}, C_{m}, \rho_{m}, \delta_{m}$.

We consider the super trace identity of super integrable systems [4] [5]

$$
\frac{\delta}{\delta u}\left(\operatorname{Str}\left(V \frac{\partial U}{\partial \lambda}\right)\right)=\lambda^{-\gamma} \frac{\partial}{\partial \lambda} \lambda^{\gamma} \operatorname{Str}\left(\frac{\partial U}{\partial u} V\right)
$$

where Str means the super trace. Defining a scalar $H=H(u, \lambda)$ by the equation

$$
H=\operatorname{Str}\left(V \frac{\partial U}{\partial \lambda}\right), \quad H=\sum_{m=0}^{\infty} H_{m}(u, \lambda) \lambda^{-m}
$$

The sets $\left\{H_{m}\right\}$ proves the conserved densities of (4). The Hmailtonian form with $H_{n+1}$ can be written as

$$
u_{t_{n}}=J \frac{\delta H_{n+1}}{\delta u}, \quad \frac{\delta H_{n}}{\delta u}=L \frac{\delta H_{n-1}}{\delta u}=\cdots=L^{n} \frac{\delta H_{0}}{\delta u}, \quad n=1,2, \cdots
$$

where $L$ is a recursion operator and $J$ is a symplectic operator, and $\frac{\delta}{\delta u}=\left(\frac{\delta}{\delta u_{1}}, \cdots, \frac{\delta}{\delta u_{p}}\right)^{\mathrm{T}}$. 
According to (3) and (5), we consider the auxiliary linear problem. For $N$ distinct $\lambda_{j}, j=1, \cdots, N$, the following systems result from (1)

$$
\begin{aligned}
& \left(\begin{array}{l}
\phi_{1 j} \\
\phi_{2 j} \\
\phi_{3 j}
\end{array}\right)_{x}=U\left(u, \lambda_{j}\right)\left(\begin{array}{l}
\phi_{1 j} \\
\phi_{2 j} \\
\phi_{3 j}
\end{array}\right)=\sum_{i=1}^{5} u_{i} e_{i}\left(\lambda_{j}\right)\left(\begin{array}{l}
\phi_{1 j} \\
\phi_{2 j} \\
\phi_{3 j}
\end{array}\right), \\
& \left(\begin{array}{c}
\phi_{1 j} \\
\phi_{2 j} \\
\phi_{3 j}
\end{array}\right)_{t_{n}}=V^{(n)}\left(u, \lambda_{j}\right)\left(\begin{array}{c}
\phi_{1 j} \\
\phi_{2 j} \\
\phi_{3 j}
\end{array}\right)=\left[\sum_{m=0}^{n} V_{m}\left(u, \lambda_{j}\right) \lambda_{j}^{n-m}+\Delta_{n}\left(u, \lambda_{j}\right)\right]\left(\begin{array}{l}
\phi_{1 j} \\
\phi_{2 j} \\
\phi_{3 j}
\end{array}\right) .
\end{aligned}
$$

Based on the results [11], we show that the following equations

$$
\frac{\delta H_{k}}{\delta u}+\sum_{j=1}^{N} \alpha_{j} \frac{\delta \lambda_{j}}{\delta u}=0
$$

where $\alpha_{j}$ are constants. Equation (11) determines a finite dimensional invariant set for the flows (9).

For (10), it is known that

$$
\frac{\delta \lambda_{j}}{\delta u}=\operatorname{Str}\left(\psi_{j} \frac{\partial U\left(u, \lambda_{j}\right)}{\partial u}\right)=\operatorname{Str}\left(\psi_{j} e_{i}\left(\lambda_{j}\right)\right), \quad i=1, \cdots, 5
$$

where Str denotes the super trace of a matrix and

$$
\psi_{j}=\left(\begin{array}{ccc}
\phi_{1 j} \phi_{2 j} & -\phi_{1 j}^{2} & \phi_{1 j} \phi_{3 j} \\
\phi_{2 j}^{2} & -\phi_{1 j} \phi_{2 j} & \phi_{2 j} \phi_{3 j} \\
\phi_{2 j} \phi_{3 j} & -\phi_{1 j} \phi_{3 j} & 0
\end{array}\right), \quad j=1, \cdots, N
$$

According to (11), for a specific $k_{0} \geq n_{0}$, we demand that

$$
\frac{\delta H_{k_{0}}}{\delta u_{i}}=\sum_{j=1}^{N} \frac{\delta \lambda_{j}}{\delta u_{i}}=\sum_{j=1}^{N} \operatorname{Str}\left(\psi_{j} e_{i}\left(\lambda_{j}\right)\right)
$$

From (9) and (11), a kind of super integrable hierarchy with self-consistent sources can be present as follows

$$
u_{i, t_{n}}=J \frac{\delta H_{n+1}}{\delta u_{i}}+J \sum_{j=1}^{N} \frac{\delta \lambda_{j}}{\delta u_{i}}=J L^{n} \frac{\delta H_{1}}{\delta u_{i}}+J \sum_{j=1}^{N} \frac{\delta \lambda_{j}}{\delta u_{i}}, \quad n=1,2, \cdots
$$

\section{The Super Tu Hierarchy with Self-Consistent Sources}

The super Tu spectral problem associated with Lie super algebra $B(0,1)$ is given by [6]

$$
\phi_{x}=U \phi, \quad U=\left(\begin{array}{ccc}
-\lambda+\frac{1}{2} q & r & \alpha \\
r & \lambda-\frac{1}{2} q & \beta \\
\beta & -\alpha & 0
\end{array}\right), \quad u=\left(\begin{array}{l}
q \\
r \\
\alpha \\
\beta
\end{array}\right), \quad \phi=\left(\begin{array}{l}
\phi_{1} \\
\phi_{2} \\
\phi_{3}
\end{array}\right)
$$

where $\lambda$ is a spectral parameter, $q$ and $r$ are even variables, $\alpha$ and $\beta$ are odd variables [6].

Taking

$$
V=\left(\begin{array}{ccc}
A & B+C & \rho \\
B-C & -A & \delta \\
\delta & -\rho & 0
\end{array}\right)
$$

The co-adjoint equation associated with (16) $V_{x}=[U, V]$ gives 


$$
\left\{\begin{array}{l}
A_{x}=-2 r C+\beta \rho+\alpha \delta \\
B_{x}=-2 \lambda C+q C-\alpha \rho+\beta \delta \\
C_{x}=-2 \lambda B-2 r A+q B-\alpha \rho-\beta \delta \\
\rho_{x}=-\lambda \rho-\alpha A-\beta B-\beta C+\frac{1}{2} q \rho+r \delta \\
\delta_{x}=\lambda \delta+\beta A-\alpha B+\alpha C+r \rho-\frac{1}{2} q \delta
\end{array}\right.
$$

If we set

$$
A=\sum_{i \geq 0} A_{i} \lambda^{-i}, \quad B=\sum_{i \geq 0} B_{i} \lambda^{-i}, \quad C=\sum_{i \geq 0} C_{i} \lambda^{-i}, \quad \rho=\sum_{i \geq 0} \rho_{i} \lambda^{-i}, \quad \delta=\sum_{i \geq 0} \delta_{i} \lambda^{-i}
$$

Then (17) is equivalent to

$$
\begin{cases}B_{i+1}=-r A_{i}+\frac{1}{2} q B_{i}-\frac{1}{2} C_{i x}-\frac{1}{2} \alpha \rho_{i}-\frac{1}{2} \beta \delta_{i}, & i \geq 0, \\ C_{i+1}=-\frac{1}{2} B_{i, x}+\frac{1}{2} q C_{i}-\frac{1}{2} \alpha \rho_{i}+\frac{1}{2} \beta \delta_{i}, & i \geq 0, \\ \rho_{i+1}=-\alpha A_{i}-\beta B_{i}-\beta C_{i}-\rho_{i, x}+\frac{1}{2} q \rho_{i}+r \delta_{i}, & i \geq 0, \\ \delta_{i+1}=-\beta A_{i}+\alpha B_{i}-\alpha C_{i}-r \rho_{i}+\frac{1}{2} q \delta_{i}+\delta_{i, x}, & i \geq 0 \\ A_{i+1, x}=-2 r C_{i+1}+\beta \rho_{i+1}+\alpha \delta_{i+1}, & i \geq 0 .\end{cases}
$$

Which results in the recurrence relations

$$
\left\{\begin{array}{l}
\left(A_{i+1}, 2 B_{i+1}, 2 \delta_{i+1},-2 \rho_{i+1}\right)^{\mathrm{T}}=L\left(A_{i}, 2 B_{i}, 2 \delta_{i},-2 \rho_{i}\right)^{\mathrm{T}}, \\
A_{i}=\partial^{-1}\left(-2 r C_{i}+\beta \rho_{i}+\alpha \delta_{i}\right), \quad i \geq 0 .
\end{array}\right.
$$

where

$$
L=\left(\begin{array}{cccc}
\frac{1}{2} \partial^{-1} q \partial & \frac{1}{2} \partial^{-1} r \partial & \frac{1}{2} \partial^{-1} \alpha \partial & \frac{1}{2} \partial^{-1} \beta \partial \\
\frac{1}{2} \partial^{-1} \frac{1}{r} \partial-2 r & \frac{1}{2} q & -\frac{1}{2} \beta-\frac{1}{4} \partial \frac{\alpha}{r} & \frac{1}{2} \alpha+\frac{1}{4} \partial \frac{\beta}{r} \\
\frac{\alpha}{r} \partial-2 \beta & \alpha & \partial+\frac{1}{2} q & r+\frac{\alpha \beta}{2 r} \\
2 \alpha-\frac{\beta}{r} \partial & \beta & -r-\frac{\alpha \beta}{2 r} & -\partial+\frac{1}{2} q
\end{array}\right)
$$

Upon choosing the initial conditions

$$
B_{0}=C_{0}=\rho_{0}=\delta_{0}=0, \quad A_{0}=1
$$

All other $A_{i}, B_{i}, C_{i}, \rho_{i}, \delta_{i}(i \geq 1)$ can be worked out by the recurrence relations (20). The first few sets are as follows:

$$
\begin{aligned}
& A_{1}=0, B_{1}=-r, C_{1}=0, \rho_{1}=-\alpha, \delta_{1}=-\beta, \\
& A_{2}=-\frac{1}{2} r^{2}-\alpha \beta, B_{2}=-\frac{1}{2} q r, C_{2}=\frac{1}{2} r_{x}, \rho_{2}=\alpha_{x}-\frac{1}{2} q \alpha, \delta_{2}=-\beta_{x}-\frac{1}{2} q \beta, \\
& A_{3}=-\frac{1}{2} q r^{2}+\alpha \beta_{x}-\alpha_{x} \beta-q \alpha \beta, B_{3}=-\frac{1}{4} r_{x x}-\frac{1}{4} q^{2} r+\frac{1}{2} r^{3}+r \alpha \beta-\frac{1}{2} \alpha \alpha_{x}+\frac{1}{2} \beta \beta_{x}, \\
& C_{3}=\frac{1}{4} q_{x} r+\frac{1}{2} q r_{x}-\frac{1}{2} \alpha \alpha_{x}-\frac{1}{2} \beta \beta_{x}, \\
& \rho_{3}=-\alpha_{x x}+\frac{1}{2} q_{x} \alpha+q \alpha_{x}-\frac{1}{4} q^{2} \alpha+\frac{1}{2} r^{2} \alpha-\frac{1}{2} r_{x} \beta-r \beta_{x}, \delta_{3}=-\beta_{x x}-\frac{1}{2} r_{x} \alpha-r \alpha_{x}-q \beta_{x}-\frac{1}{4} q^{2} \beta+\frac{1}{2} r^{2} \beta-\frac{1}{2} q_{x} \beta .
\end{aligned}
$$

Let us associate the problem (16) with the following auxiliary problem 


$$
\phi_{t_{n}}=V^{(n)} \phi, \quad V^{(n)}=\sum_{i=0}^{n}\left(\begin{array}{ccc}
A_{i} & B_{i}+C_{i} & \rho_{i} \\
B_{i}-C_{i} & -A_{i} & \delta_{i} \\
\delta_{i} & -\rho_{i} & 0
\end{array}\right) \lambda^{n-i}+\left(\begin{array}{ccc}
\frac{B_{n+1}}{r} & 0 & 0 \\
0 & -\frac{B_{n+1}}{r} & 0 \\
0 & 0 & 0
\end{array}\right)
$$

The compatible conditions of the spectral problem (16) and the auxiliary problem (22) are

$$
U_{t_{n}}-V_{x}^{(n)}+\left[U, V^{(n)}\right]=0
$$

Which refer the super Tu equation hierarchy

$$
u_{t_{n}}=K_{n}=\left(2\left(\frac{B_{n+1}}{r}\right)_{x},-2 C_{n+1},-\rho_{n+1}+\frac{\alpha}{r} B_{n+1}, \delta_{n+1}-\frac{\beta}{r} B_{n+1}\right)^{\mathrm{T}} .
$$

Here $u_{t_{n}}=K_{n}$ in (24) is called the $n$-th Tu flow of this hierarchy.

Using the super trace identity (7), we have

$$
\left(\begin{array}{llll}
A_{i+1} & 2 B_{i+1} & 2 \delta_{i+1} & -2 \rho_{i+1}
\end{array}\right)^{\mathrm{T}}=\frac{\delta}{\delta u} H_{i}, \quad H_{i}=\int \frac{2 A_{i+2}}{i+1} \mathrm{~d} x, \quad i \geq 0
$$
form:

Therefore, the super Tu soliton hierarchy Equation (24) can be written as the following super Hamiltonian

$$
u_{t_{n}}=J \frac{\delta H_{n}}{\delta u}
$$

where

$$
J=\left(\begin{array}{cccc}
0 & \partial \frac{1}{r} & 0 & 0 \\
\partial \frac{1}{r} & 0 & -\frac{\alpha}{2 r} & -\frac{\beta}{2 r} \\
0 & \frac{\alpha}{2 r} & 0 & \frac{1}{2} \\
0 & \frac{\beta}{2 r} & \frac{1}{2} & 0
\end{array}\right)
$$

Is a super symplectic operator, and $H_{n}$ is given by (25).

The first non-trivial nonlinear of super Tu hierarchy is given by its second flow

$$
\left\{\begin{array}{l}
q_{t_{2}}=-\frac{1}{2}\left(\frac{r_{x x}}{r}\right)_{x}-q q_{x}+2 r r_{x}+2(\alpha \beta)_{x}-\left(\frac{\alpha \alpha_{x}}{r}\right)_{x}+\left(\frac{\beta \beta_{x}}{r}\right)_{x}, \\
r_{t_{2}}=-\frac{1}{2} q_{x} r-q r_{x}+\frac{1}{2} \alpha \alpha_{x}+\beta \beta_{x}, \\
\alpha_{t_{2}}=\alpha_{x x}-\frac{1}{2} q_{x} \alpha-q \alpha_{x}-r^{2} \alpha+\frac{1}{2} r_{x} \beta+r \beta_{x}-\frac{r_{x x}}{4 r} \alpha+\frac{1}{2 r} \alpha \beta \beta_{x}, \\
\beta_{t_{2}}=-\beta_{x x}-\frac{1}{2} r_{x} \alpha-r \alpha_{x}-q \beta_{x}+r^{2} \beta-\frac{1}{2} q_{x} \beta+\frac{r_{x x}}{4 r} \beta+\frac{1}{2 r} \alpha \alpha_{x} \beta .
\end{array}\right.
$$

Which possesses a Lax pair of $U$ defined in (16) and $V^{(2)}$ defined by

$$
V^{(2)}=\left(\begin{array}{ccc}
\lambda^{2}-\frac{r_{x x}}{4 r}-\frac{1}{4} q^{2}-\frac{\alpha \alpha_{x}}{2 r}+\frac{\beta \beta_{x}}{2 r} & -r \lambda-\frac{1}{2} q r+\frac{1}{2} r_{x} & -\alpha \lambda+\alpha_{x}-\frac{1}{2} q \alpha \\
-r \lambda-\frac{1}{2} q r-\frac{1}{2} r_{x} & -\lambda^{2}+\frac{r_{x x}}{4 r}+\frac{1}{4} q^{2}+\frac{\alpha \alpha_{x}}{2 r}-\frac{\beta \beta_{x}}{2 r} & -\beta \lambda-\beta_{x}-\frac{1}{2} q \beta \\
-\beta \lambda-\beta_{x}-\frac{1}{2} q \beta & \alpha \lambda-\alpha_{x}+\frac{1}{2} q \alpha & 0
\end{array}\right)
$$


Next we will establish the super Tu hierarchy with self-consistent sources. Consider the linear system

$$
\left(\begin{array}{l}
\phi_{1 j} \\
\phi_{2 j} \\
\phi_{3 j}
\end{array}\right)_{x}=U\left(\begin{array}{l}
\phi_{1 j} \\
\phi_{2 j} \\
\phi_{3 j}
\end{array}\right)=\left(\begin{array}{ccc}
-\lambda+\frac{1}{2} q & r & \alpha \\
r & \lambda-\frac{1}{2} q & \beta \\
\beta & -\alpha & 0
\end{array}\right)\left(\begin{array}{l}
\phi_{1 j} \\
\phi_{2 j} \\
\phi_{3 j}
\end{array}\right), \quad\left(\begin{array}{l}
\phi_{1 j} \\
\phi_{2 j} \\
\phi_{3 j}
\end{array}\right)_{t_{n}}=V\left(\begin{array}{l}
\phi_{1 j} \\
\phi_{2 j} \\
\phi_{3 j}
\end{array}\right)=\left(\begin{array}{ccc}
A & B+C & \rho \\
B-C & -A & \delta \\
\delta & -\rho & 0
\end{array}\right)\left(\begin{array}{l}
\phi_{1 j} \\
\phi_{2 j} \\
\phi_{3 j}
\end{array}\right)
$$

For the system (28), we consider the $\frac{\delta H}{\delta u}=\sum_{j=1}^{N} \frac{\delta \lambda_{j}}{\delta u}$ in the Lie super algebra $B(0,1)$ and obtain

$$
\frac{\delta \lambda_{j}}{\delta u}=\left(\begin{array}{l}
\operatorname{Str}\left(\Psi_{j} \frac{\partial U}{\partial q}\right) \\
\operatorname{Str}\left(\Psi_{j} \frac{\partial U}{\partial r}\right) \\
\operatorname{Str}\left(\Psi_{j} \frac{\partial U}{\partial \alpha}\right) \\
\operatorname{Str}\left(\Psi_{j} \frac{\partial U}{\partial \beta}\right)
\end{array}\right)=\left(\begin{array}{c}
\left\langle\Phi_{1}, \Phi_{2}\right\rangle \\
\left\langle\Phi_{2}, \Phi_{2}\right\rangle-\left\langle\Phi_{1}, \Phi_{1}\right\rangle \\
-2\left\langle\Phi_{2}, \Phi_{3}\right\rangle \\
2\left\langle\Phi_{1}, \Phi_{3}\right\rangle
\end{array}\right)
$$

where $\Phi_{i}=\left(\phi_{i 1}, \cdots, \phi_{i N}\right)^{\mathrm{T}}(i=1,2,3)$.

According to the results in (15), the super Tu hierarchy with self-consistent sources is presented as

$$
u_{t_{n}}=\left(\begin{array}{c}
q \\
r \\
\alpha \\
\beta
\end{array}\right)_{t_{n}}=J L^{n}\left(\begin{array}{c}
0 \\
-2 r \\
-2 \beta \\
2 \alpha
\end{array}\right)+J\left(\begin{array}{c}
\left\langle\Phi_{1}, \Phi_{2}\right\rangle \\
\left\langle\Phi_{2}, \Phi_{2}\right\rangle-\left\langle\Phi_{1}, \Phi_{1}\right\rangle \\
-2\left\langle\Phi_{2}, \Phi_{3}\right\rangle \\
2\left\langle\Phi_{1}, \Phi_{3}\right\rangle
\end{array}\right)
$$

The first nontrivial integrable super Tu hierarchy with self-consistent sources is its second flow

$$
\left\{\begin{array}{l}
q_{t_{2}}=-\frac{1}{2}\left(\frac{r_{x x}}{r}\right)_{x}-q q_{x}+2 r r_{x}+2(\alpha \beta)_{x}-\left(\frac{\alpha \alpha_{x}}{r}\right)_{x}+\left(\frac{\beta \beta_{x}}{r}\right)_{x}+\left(\frac{\left\langle\Phi_{2}, \Phi_{2}\right\rangle-\left\langle\Phi_{1}, \Phi_{1}\right\rangle}{r}\right)_{x}, \\
r_{t_{2}}=-\frac{1}{2} q_{x} r-q r_{x}+\frac{1}{2} \alpha \alpha_{x}+\beta \beta_{x}+\left(\frac{\left\langle\Phi_{1}, \Phi_{2}\right\rangle}{r}\right)_{x}+\frac{\alpha}{r}\left\langle\Phi_{2}, \Phi_{3}\right\rangle+\frac{\beta}{r}\left\langle\Phi_{1}, \Phi_{3}\right\rangle, \\
\alpha_{t_{2}}=\alpha_{x x}-\frac{1}{2} q_{x} \alpha-q \alpha_{x}-r^{2} \alpha+\frac{1}{2} r_{x} \beta+r \beta_{x}-\frac{r_{x x}}{4 r} \alpha+\frac{1}{2 r} \alpha \beta \beta_{x}+\frac{\alpha}{2 r}\left(\left\langle\Phi_{2}, \Phi_{2}\right\rangle-\left\langle\Phi_{1}, \Phi_{1}\right\rangle\right)+\left\langle\Phi_{1}, \Phi_{3}\right\rangle, \\
\beta_{t_{2}}=-\beta_{x x}-\frac{1}{2} r_{x} \alpha-r \alpha_{x}-q \beta_{x}+r^{2} \beta-\frac{1}{2} q_{x} \beta+\frac{r_{x x}}{4 r} \beta+\frac{1}{2 r} \alpha \alpha_{x} \beta-\frac{\beta}{2 r}\left(\left\langle\Phi_{2}, \Phi_{2}\right\rangle-\left\langle\Phi_{1}, \Phi_{1}\right\rangle\right)-\left\langle\Phi_{2}, \Phi_{3}\right\rangle .
\end{array}\right.
$$

When $\alpha=\beta=0$, it is the well known nonlinear Tu equation with self-consistent sources. So system (30) is a novel super integrable equation hierarchy.

\section{Conservation Laws for the Super Tu Hierarchy}

In what follows, we will construct conservation laws of the super coupled Burgers equation. Introduce the variables:

$$
K=\frac{\phi_{2}}{\phi_{1}}, \quad G=\frac{\phi_{3}}{\phi_{1}},
$$

where $p(K)=0, p(G)=1$. From (10), we have

$$
K_{x}=r+(2 \lambda-q) K+\beta G-r K^{2}-\alpha K G, \quad G_{x}=\beta-\alpha K+\left(\lambda-\frac{1}{2} q\right) G-r K G-\alpha G^{2}
$$

We expand $K, G$ in powers of $\lambda^{-1}$ as follows 


$$
K=\sum_{j=0}^{\infty} k_{j} \lambda^{-j}, \quad G=\sum_{j=0}^{\infty} g_{j} \lambda^{-j},
$$

where $p\left(k_{j}\right)=0, p\left(g_{j}\right)=1$. Substituting (34) into (33) and comparing the coefficients of the same powers of $\lambda$, we obtain

$$
\begin{aligned}
& k_{1}=-\frac{1}{2} r, g_{1}=-\beta, k_{2}=-\frac{1}{4} r_{x}-\frac{1}{4} q r, g_{2}=-\beta_{x}-\frac{1}{2} r \alpha-\frac{1}{4} q r, \\
& k_{3}=-\frac{1}{8} r_{x}-\frac{1}{8} q_{x} r-\frac{1}{4} q r_{x}-\frac{1}{8} q^{2} r+\frac{1}{2} \beta \beta_{x}+\frac{1}{8} q r \beta+\frac{1}{8} r^{3}, \\
& g_{3}=-\beta_{x x}-\frac{3}{4} r_{x} \alpha-\frac{1}{2} q r \alpha-\frac{1}{2} r \alpha_{x}-\frac{1}{4} q_{x} r-\frac{1}{4} q r_{x}-\frac{1}{2} q \beta_{x}-\frac{1}{8} q^{2} r+\frac{1}{2} r^{2} \beta .
\end{aligned}
$$

And a recursion formula for $k_{n}$ and $g_{n}$,

$$
\begin{aligned}
& k_{n+1}=\frac{1}{2} k_{n x}+\frac{1}{2} q k_{n}-\frac{1}{2} \beta g_{n}+\frac{1}{2} r\left(\sum_{l=0}^{n} k_{l} k_{n-l}\right)+\frac{1}{2} \alpha\left(\sum_{l=0}^{n} k_{l} g_{n-l}\right), \\
& g_{n+1}=g_{n x}+\alpha k_{n}+\frac{1}{2} q g_{n}+r\left(\sum_{l=0}^{n} k_{l} g_{n-l}\right)+\alpha\left(\sum_{l=0}^{n} g_{l} g_{n-l}\right) .
\end{aligned}
$$

Because of

$$
\frac{\partial}{\partial t} \frac{\phi_{1, x}}{\phi_{1}}=\frac{\partial}{\partial x} \frac{\phi_{1, t}}{\phi_{1}}
$$

we derive the conservation laws of (27)

$$
\frac{\partial}{\partial t}\left(-\lambda+\frac{1}{2} q+r K+\alpha G\right)=\frac{\partial}{\partial x}(A+(B+C) K+G)
$$

where

$$
\begin{aligned}
& A=c_{0} \lambda^{2}+c_{1} \lambda-\frac{1}{2} c_{0} r^{2}-c_{0} \alpha \beta, \\
& B=-c_{0} r \lambda-c_{1} r-\frac{1}{2} c_{0} q r, \\
& C=\frac{1}{2} c_{0} r_{x}, \\
& \rho=-c_{0} \alpha \lambda-c_{1} \alpha+c_{0} \alpha_{x}-\frac{1}{2} c_{0} q \alpha .
\end{aligned}
$$

Assume that $\sigma=-\lambda+\frac{1}{2} q+r K+\alpha G, \theta=A+(B+C) K+\rho G$, then (38) can be written as $\sigma_{t}=\theta_{x}$, which is the right form of conservation laws. We expand $\sigma$ and $\theta$ as series in powers of $\lambda$ according with the coefficients, which are called conserved densities and currents respectively

$$
\sigma=-\lambda+\sum_{j=0}^{\infty} \sigma_{j} \lambda^{-j}, \quad \theta=c_{0} \lambda^{2}+c_{1} \lambda+\sum_{j=0}^{\infty} \theta_{j} \lambda^{-j}
$$

where $c_{0}, c_{1}$ are constants of integration. Then the first two conserved densities and currents are

$$
\begin{aligned}
\sigma_{1}= & -\frac{1}{2} r^{2}-\alpha \beta, \\
\sigma_{2}= & -\frac{1}{4} r r_{x}-\frac{1}{4} q r^{2}-\alpha \beta_{x}-\frac{1}{4} q r \alpha, \\
\theta_{1}= & c_{0}\left(\frac{1}{2} q r^{2}+\alpha \beta_{x}+\frac{1}{4} q r \alpha-\alpha_{x} \beta+\frac{1}{2} q \alpha \beta\right)+c_{1}\left(\frac{1}{2} r^{2}+\alpha \beta\right), \\
\theta_{1}= & c_{0}\left(\frac{1}{8} r r_{x}+\frac{1}{8} q_{x} r^{2}+\frac{1}{4} q r r_{x}+\frac{1}{4} q^{2} r^{2}-\frac{1}{2} r \beta \beta_{x}-\frac{1}{8} q r^{2} \beta-\frac{1}{8} r^{4}+\alpha \beta_{x x}+r \alpha \alpha_{x}\right. \\
& \left.+\frac{1}{4} q_{x} r \alpha+\frac{1}{4} q r_{x} \alpha+q \alpha \beta_{x}+\frac{1}{8} q^{2} r \alpha-\frac{1}{2} r^{2} \alpha \beta-\alpha_{x} \beta_{x}+\frac{1}{8} q r \alpha\right)+c_{1}\left(\frac{1}{4} r r_{x}+\frac{1}{4} q r^{2}+\alpha \beta_{x}+\frac{1}{4} q r \alpha\right) .
\end{aligned}
$$


The recursion relations for $\sigma_{n}$ and $\theta_{n}$ are

$$
\sigma_{n}=r k_{n}+\alpha g_{n}, \quad \theta_{n}=c_{0}\left(-r k_{n+1}-\frac{1}{2} q r k_{n}+\frac{1}{2} r_{x} k_{n}-\alpha g_{n+1}-\alpha_{x} g_{n}-\frac{1}{2} q \alpha g_{n}\right)+c_{1}\left(-r k_{n}-\alpha g_{n}\right)
$$

where $k_{n}$ and $g_{n}$ can be calculated from (36). The infinitely conservations laws of (36) can be easily obtained in (32)-(40) respectively.

\section{Acknowledgements}

This work was supported by the Natural Science Foundation of Henan Province (No. 132300410202), the Science and Technology Key Research Foundation of the Education Department of Henan Province (No. 12A110017, 14A110010), the Youth Backbone Teacher Foundation of Shangqiu Normal University (No. 2013GGJS02).

\section{References}

[1] Ma, W.X. and Strampp, W. (1994) An Explicit Symmetry Constraint for the Lax Pairs of AKNS Systems. Physics Letters A, 185, 277-286. http://dx.doi.org/10.1016/0375-9601(94)90616-5

[2] Ma, W.X. (2003) Soliton, Positon and Negaton Solutions to a Schrödinger Self-Consistent Source Equation. Journal of the Physical Society of Japan, 72, 3017-3019. http://dx.doi.org/10.1143/JPSJ.72.3017

[3] Ma, W.X. and Geng, X.G. (2001) Bäcklund Transformations of Soliton Systems from Symmetry Constraints. CRM Proceedings and Lecture Notes, 29, 313-323.

[4] Hu, X.B. (1997) An Approach to Generate Superextensions of Integrable Systems. Journal of Physics A: Mathematical and General, 30, 619-632. http://dx.doi.org/10.1088/0305-4470/30/2/023

[5] Ma, W.X., He, J.S. and Qin, Z.Y. (2008) A Supertrace Identity and Its Applications to Super Integrable Systems. Journal of Mathematical Physics, 49, Article ID: 033511. http://dx.doi.org/10.1063/1.2897036

[6] Wang, X.Z. and Liu, X.K. (2010) Two Types of Lie Super-Algebra for the Super-Integrable Tu-Hierarchy and Its SuperHamiltonian Structure. Communications in Nonlinear Science and Numerical Simulation, 15, 2044-2049. http://dx.doi.org/10.1016/j.cnsns.2009.09.008

[7] Yu, J., Han, J.W. and He, J.S. (2009) Binary Nonlinearization of the Super AKNS System under an Implicit Symmetry Constraint. Journal of Physics A: Mathematical and Theoretical, 42, Article ID: 465201. http://dx.doi.org/10.1088/1751-8113/42/46/465201

[8] Yu, J., He, J.S., Ma, W.X. and Cheng, Y. (2010) The Bargmann Symmetry Constraint and Binary Nonlinearization of the Super Dirac System. Chinese Annals of Mathematics, Series B, 31, 361-372. http://dx.doi.org/10.1007/s11401-009-0032-6

[9] Liu, Q.P. (1995) Darboux Transformations for Supersymmetric Korteweg-De Equations. Letters in Mathematical Physics, 35, 115-122. http://dx.doi.org/10.1007/BF00750761

[10] Siddiq, M., Hassan, M. and Saleem, U. (2006) On Darboux Transformation of the Supersymmetric Sine-Gordon Equation. Journal of Physics A: Mathematical and General, 39, 7313-7318. http://dx.doi.org/10.1088/0305-4470/39/23/010

[11] Zeng, Y.B., Ma, W.X. and Lin, R.L. (2000) Integration of the Soliton Hierarchy with Self-Consistent Sources. Journal of Mathematical Physics, 41, 5453-5489. 